\title{
Issues of Sex and Sexuality: Reflections on Persons with Disabilities.
}

\author{
Dhemba Ishmael $^{1}$, Dhemba Regina ${ }^{2}$ \\ ${ }^{1}$ Lecturer - Southern Africa Nazarene University (SANU) - (Special and Inclusive Education Department) - \\ Swaziland. \\ ${ }^{2}$ Trainer - EduGate Training Centre - Swaziland.
}

\begin{abstract}
Realities of Sex and sexuality show that this is a subject that has been talked about in harsh tones, especially in some greater parts of African communities. Having or not having sex, sex is a term that is well known, yet it has been given very little attention when it comes to serious public discussions and debates. One of the reasons being that it is still rather a taboo, in African traditional and modern communities, alike, to publicly discuss sex related topics since they are loosely connected to genitalia or bedroom activity. While sexuality, as a term that generally defines capacity for sexual feelings, includes those body parts not commonly discussed, it has to be known that, the term is constitutive of all which define who we are, in terms of the way we behave and/or react like: speak, smile, laugh, dress, or generally carry ourselves as expressed through gait or posture of standing, sitting or walking. So, whether we talk about it, shun or refrain from the topic or subject, we still remain influential on the sexuality of others, especially our children, inclusive of those with disabilities. It is for that reason that there is need to openly talk about sex and sexuality related issues.
\end{abstract}

Keywords: Cultural beliefs, Culture, Disability, Exclusion, Hand job, Impairment(s), Inclusion, Myth(s), Sex, Sexuality

\section{INTRODUCTION}

Sex and sexuality are terms which have to be known and appropriately used, both in theory and practice, since they border around life and living. This remains so, notwithstanding the truth that these terms are surrounded with myths, especially if they relate to persons with disabilities. In the African context, for example, a subject area with sexual functioning content was, and is still considered culturally inappropriate to open discussions/debates, and hence assumed to be outside the domains and scope of those persons with disabilities' area of legitimate concern. Even today, a lot of misunderstandings and misconceptions around the subject of sex and sexuality seem to carry a reality that is detrimental to the life and general welfare of persons with disabilities.

\section{Understanding disability, sex, sexuality and culture}

The scope of disability has been known to be quite wide, rendering it problematic to even define. However, Chakuchichi, Mapepa and Mutasa (2010) are quoted as saying that the term disability denotes lack of ability to perform some desired functions. In its literal sense, disability implies a deprivation or loss of the needed competency, as it imposes limitations on overall development, especially on skill(s) acquisition. Part of the implication of this proposition may suggest that one is classified as having a disability if they have any form of impairment(s). Having a disability or not, the subject of sex and sexuality has to carry an inclusive mandate in today's societal debates and discussions. The term 'sexuality', as found in http://www. scarleteen.com/article/sexuality, has been used and misused a lot, just like the word 'sex.' These two terms, sex and sexuality, have been heard and referred to a lot, yet are often not clearly defined, at times.

In order to understand issues of sexuality, therefore, it is important to clearly understand some very basic information about the term sex, a term that has always been narrowly characterised to signify penis-invagina action or anal penetration. This is a seriously warped understanding of the term sex, since there are many people who don't or can't have that kind of genital sex, but still have active, fulfilling sex lives through other means, like autoeroticism or such autosexual oriented acts, including the current day intensive publicity and hype for use of phone sex.

Apart from kissing and frottage, where partners rub their bodies against each other, usually with clothes on, for example, other sexual fulfillments also come through manual sex, that come in all shades and colour like: sexual massage, fingering, petting, stroking, breast or nipple stimulation, intrapersonal masturbation, mutual masturbation and all sort of handjobs, which, according to www.yourdictionary.com is a vulgar slang term for the action of a person stimulating self or another. Illustratively, just one example of handjobs is like an action 
when a woman uses her hands, on a man's penis, in order for the man to reach a climax of sexual excitement orgasm (Sexual Resource Center for Parents - [SRCP], 2017).

Notwithstanding the fact that sexuality is a term that also includes body parts, like in the term sex, sexuality is, however, more than awareness and mutual utilisation of these body parts. In its broad sense, sexuality, as SRCP (2017) defines, includes one's gender identity, which is the core sense that we are either female/male or gender roles, which is the idea of how we should behave because we are a female or male. Sexuality also includes people's sexual orientation, as characterised by being either heterosexual, homosexual, or bisexual (SRCP, 2017). Impressions emerging from the above seem to suggest that sexuality is an integral part of our personal selves, in terms of: who we are, our feelings, our beliefs and everything that surround our intrapersonal and interpersonal relationships. Such information is important to all people, with or without disabilities.

\section{Psychological and Physiological perspectives on sexuality}

Mutasa and Tafangombe (2010) assert that sexuality can be viewed from both psychological and physiological perspectives. While, on the one hand, the psychological perspective sees sexuality as an expression of love, through affection and intimacy, physiologically, on the other hand, takes sexuality as a function of personality and emotions, as it relates to sexual arousal and behaviours. Giving it a cultural touch, sexuality has been understood as the combination of sex drive, sex acts and all those aspects of personality concerned with learned communication and relationship patterns, as determined by a culture of a defined society or community, as Merinelli and DellOrto (1984) augments.

Although issues of genitals, penetration and/or all those sexual pleasures seem to quickly come to one's mind, on first mention of the term 'sexuality', the above understanding has shown that the subject of sexuality is much broader than sex, which is only a small part that characterizes who we are. If sexuality refers to those things people think, feel and do, as related to their sexual orientations, sexual patterns, roles and desires, over and above just the capacity for sexual feelings, then there is that very great need for every human being, with or without a disability to clearly understand the principles of sexuality, in its truest sense.

\section{Public and Private sexuality to persons with disabilities}

In a way of clarifying issues of sexuality, Arnot and Weiner (1987) \& Barron and Norris (1998) put sexuality into two broad categories of Public Sexuality and Private Sexuality.

\section{(i) Public sexuality}

On the one hand, public sexuality is concerned with how a person acts in public, and the role played by those actions. Sexual patterns and roles are our public demonstration of socially recognised sexual expressions. Public concerns may manifest themselves in the choice of how the individuals interact with society. In the domains of this paper, Public sexuality, therefore, grapples with a question like: Will a disability interfere with the individual's personal or public appraisal of their masculinity or feminity? That's the question to unravel or disentangle.

\section{(ii) Private sexuality}

On the other hand, private sexuality refers to the genital, sexual responses and those inner issues not usually perceptible or discernible. Topical matters in private sexuality, as Barron and Norris (1998) put it, may include issues like the ability or inability to maintain an erection, have orgasm, receive and give genital and sensual pleasures by different means, like the use of the finger technique to blow a partner's mind, or all sorts of those handjobs, raised earlier, somewhere in this paper. However, if a partner finds the finger use to be lovingly tactile and a physically pleasing form of overwhelmingly impressive sex, for example, knowledge of sexuality has to bring an understanding that when a gynecologist does the same finger use on genitals, during a medical examination, that is not private sexuality, for none would be gratified by such, as SRCP (2017) puts it. Same goes with kissing, which is understood as sex when you lovingly kiss your partner, yet in sexuality jargon, kissing is not sex when, for example, you kiss a visiting aunt. So, we need to be mindful of the context in issues of sex and private sexuality. Let it be known that private sexuality also includes that ability to reduce or control sexual tension in oneself or partner. So, depriving persons with disabilities of all this information is truly showing faulty judgment or reasoning by the mainstream community - misguided, to say the least!

As may be noted above, be it public or private, sexuality is known as a combination of sex drive, sex acts, and all those aspects of personality concerned with relationship patterns. Such are issues which deserve very open discussions with all people, irrespective of their body condition(s), since they define our lives as sexual beings. Unfortunately, like has been raised somewhere in this paper, topics to do with sex and sexuality are issues which have not been freely discussed with persons with disabilities, especially in most African 
communities. A number of reasons may be attributable to this, one of them being that of cultural beliefs and practices or just the general misunderstandings of the whole subject of sex and sexuality.

If culture is considered as a set of ideas, beliefs and a way of behaviour of a particular organisation or group of people/society, if Haralambos and Holborn's (1990) assertion is anything to go by, then some of the misconceptions found in the subject of sex and sexuality hinge in culture of a people, and hence socially constructed. An example that quickly comes into mind is that with some cultures, persons with disabilities were viewed or generally assumed to be sexually dysfunctional, a notion that seems to still prevail even today, in some parts of human societies. This remains so, notwithstanding a truism by Mutasa and Tafangombe (2010), that persons with disabilities are also confronted with a similar variety of sexual stimuli and sexual interest, phenomena which have been known to occur at approximately the same age as for those with no disabilities.

The above may further be consolidated by these writers' interpersonal connections with persons with disabilities, where it was established that there were more similarities than differences, on sexual realities, as encountered by both the able bodied and those persons with disabilities. Through casual talk with, and observations of these people with disabilities, it was learnt that although certain disabilities may impose some differences and limitations, the reality of the matter was that facts surrounding sex and sexuality apply to those with disabilities in the same way as they do to those with no disabilities. This said, the differences commonly referred to by people are, therefore, more of imposition than reality, just used as factors to confirm that a disability comes with wide degree of negative effects. Since the biggest sex organ of the human body is the brain, these persons with disabilities also want to cruise around in the mystical world of sexual fantasy, as the brain wanders (Barron and Norris, 1998). If only included, those with disabilities would also achieve sex drives through normal platforms like: talking in sexual ways, sharing sexual fantasies, sexual role-playing, etc., as either taken from past experiences or through imagination. A puzzle that we may want to unravel or unscramble here is: Why are people with disabilities, therefore, not openly engaged in discussions related to matters of sex and sexuality?

Probably a functionalist perspective may be used to try and untangle this puzzle. Recorded African histories, for example, show that culture has always placed great importance to individual's personal contribution(s) to social equilibration. For this reason, it was, therefore, believed that as one develops a disability, they become a missing link to complete a social fabric. To further reflect on this belief, reference is made to Barron and Norris's (1998) medical perspective model research whose findings were that many African communities have a distorted and hence a warped belief that people with disabilities are mere numbers than members of a given community, since they were viewed as too sick to contribute anything to the stability of the society. In practice, these communities have a wrong notion that since these persons have one disability or the other, with some of them not being intellectual giants, they, however, were no dopes. Considering them stupid, because of the nature of their disability, only renders an unfortunate justification for these people's exclusion in important or topical issues of sex and sexuality. As equal human beings, the mainstream communities forget that even with a disability, these persons have to also enjoy the same rights and privileges accorded to every other sexual human being, in the mainstream society. With a lot of these unfounded cultural reasons, which are predominantly exclusionary in nature, persons with disabilities' knowledge on sexuality and sexual functions remain totally obscured.

\section{Stable Sexual Identity}

Some communities seem to forget or deliberately ignore the truism that every child, especially at adolescence, has a problem of attaining a stable sexual identity. To the sighted children/adolescents, for example, there are normal opportunities for sexual exploration, as they enjoy exploring sexual body parts through the normal course of play. Through such Montessori play-way method of learning, characterised by self-directed activity and collaborative play, these agers show their keen interest of the opposite sex by observing others, including the sexual organs of those peers and playmates. The sighted learn and become aware of the other's body parts by mere looking or observing. Unlike the sighted who have the privilege to see and/or observe even from a distance, those with congenital total blindness, for example, will never experience such an 'opportunity', hence the need for them to be manually taught in order for them to explore those sexual body parts.

From the above, it may be learnt that important sex and sexuality information may also be acquired by teaming up with age mates through role-plays, which, according to Mutasa and Tafangombe (2010) teach norms and acceptable behaviours, inclusive of sexual orientation preferences within heterosexual and homosexual relationships. Such gathering and role-plays are significant in developing one's sexual culture through play-way methods. Such knowledge exposes these people to acceptable global sexual practices to adopt, as characterised by intimacy, affection and love; if the psychological perspective of sexuality is anything to go by.

At this level, children are also exposed to gender beliefs which emphasize passivity for women and assertiveness for men, in every domain of life, inclusive of sexual encounters, where men are stereotyped as 
sexually overactive while women are less so, or such uncouth stereotypical beliefs. Since behaviours on sexuality are not innate, but learnt and developed through socialisation processes, it may, therefore, be realized how damaging the African culture, for example, has been, when sidelining person with disabilities from issues to do with sex and sexuality.

Unfortunately, because of some of those long standing uncouth traditional beliefs in some communities, which still take a disability as contagious, those with sensory impairments which are visual, auditory or a combination thereof, in nature, have particular difficulties, especially in learning sexual functions. No one is prepared to seriously manually engage the totally blind and/or the deaf-blind, for example, in such important discussions. Those with hearing impairment will feel the discomfort as they miss out on the general discussions in their immediate environment, which, due to the sensitivity of the subject, normally take place in whispers. From such goings on, a need should always be there to totally defy these discriminatory and exclusionary cultural practices, and embrace human diversity by truly including persons with disabilities to issues about sex and sexuality in homes, work places, at school and all such placements.

If anything, the discussed exclusionary practices are detrimental to the socialisation process of persons with disabilities. Association deprivation prevents children from acquiring the much needed information about sex and sexuality. Because of some exclusionary beliefs and practices, which have been known to be cultural, at times, the orthopedically impaired, for example, also miss out a lot due to their mobility challenges, which deny them the opportunity to access all spaces and places which their age mates visit, during play, without expenditure of much energy (Mutasa and Tafangombe, 2010). It's very unfortunate that with any form of disability, some known boorish age-old beliefs are that such people do not need information that borders around sex and sexuality.

\section{Sex as a primary drive}

Some communities' beliefs and cultural practices tend to forget some very important facts about sex which are: It is one of the four primary drives, the others being hunger, thirst, and avoidance of pain (Bernard, 2005). These drives are applicable to everyone, including those with disabilities. The other important issue forgotten by some traditional communities, if the physiological perspective of sexuality is anything to go by, is that pleasure and sometimes the desire to reproduce, are reasons why people have sex. Although these are innate conditions, certain sexual feelings, decisions and behaviours need to be controlled (Firestone, 2001). Unfortunately, persons with disabilities are ordinarily left out in matters to do with sex and sexuality, like birth control, a medical innovation that has greatly impacted, either positively or negatively to human kind population.

It is unfortunate that some sections of human kind have maintained a belief that a disability correlates non-sexuality, hence the prevalence of some sexuality prohibitory practices in some communities, which have not only discriminated, but excluded persons with disabilities. In order to take comfort in excluding them, persons with disabilities have been made to believe that the use of contraception, for birth control does not only call for the wrath of the ancestors, but is also sinful and ungodly. Such myths and unfounded reasons surrounding sex and sexuality have been used; not only to justify reasons for denying persons with disabilities access to birth control information, for example, but to typically deter them, in order to successfully keep these persons with disabilities away from the much needed sex and sexuality education, for fear of instant or aftermath consequences. At the end of the day, such vital information remains a preserve for the non-disabled population, as if those with disabilities do not have full control over their bodies. This has been known as one of the reasons why persons with disabilities end up having blotted or large numbers of children, who, in most cases are very closely spaced. As if they did not have full control over their lives, experience working with persons with disabilities has shown that they sometimes had other people to do the thinking for them, as they are always told what is good or bad for them. By not giving them an opportunity to think for themselves and decide for their bodies, these persons' very private life is constantly monitored. It is unfortunate that even today, some communities still do not think of persons with disabilities as being capable of doing anything, not only independently, but privately as well.

The culture of social isolation has also been harboured by the medical personnel. For many years, some communities have always felt that persons with disabilities should be restrained from sexual activity, lest they procreate children they cannot raise. Perturbingly, some health personnel have even gone to an extent of administering some medication and/or drugs that prevent persons with disabilities from conceiving. What made the whole experience even worse is that the administration of such drugs to those with disabilities was effected without one's consent; an approach social justice will never embrace. Since they were considered asexual, people with disabilities were discouraged from marrying for the purpose of reproducing: In fact, with a disability, one would be discouraged from leading a normal sexual life. The over preached human rights seem not to apply, when it comes to people with disabilities. 
While sexuality includes how we feel about our bodies, parents in some of these African societies have been known to have seriously contributed to their children's poor body image, by making them lead a sedentary life style. Poor body image may have a profound effect on the person with disability's ability to developing healthy sexual relationships, since they will be seen as sexually unattractive. With a disability, coupled with a poor body image, one will not have a good partner. By developing negative or poor self-images, some of these children ended up being abused, since they will not have full information relating to sex and sexuality. For lack of knowledge, if the proverbial saying is anything to go by, those with disabilities will always be abusers in the name of sexuality. Because they will lack proper knowledge about sex and sexuality issues, these children will not understand when to tolerate a touch or a pat, and when such would be more of sexual abuse than an expression of sexuality. Such confusion comes because sexuality also includes how we experience intimacy, touch, love, etc., characteristics which are also found in sex overtures.

In some communities today, young children and adolescence with disabilities are at great risk of being sexually abused by terminally ill people, with the belief that there will be an eminent cure of particular illnesses, especially if one with a terminal disease had sexual intercourse with a person with a disability or other medical condition like albinism. For lack of proper knowledge, some persons with disabilities can even be raped and enticed into believing that the sexual assault was consensual. These persons will never be privileged to understanding that although vaginal or other forms of sexual penetration may be evident, rape is not sex! On this note, Heather Corinna in SRCP (2017) further cautions by saying that it's only sex if both people want it, and when talking about sex, it is consensual sex being referred to.

If one has sex with oneself, by responding fairly well to touch of self erotically, that shouldn't be a problem. It is still not a problem if one self gratifies with some gently strokes or caress, as a sign of autoeroticism and/or some form of autosexual orientations, over other forms of sexual activity (Apfelbaum, 1989). But when you are having sex with someone or other people even, it should be sex that is wanted and freely chosen by everyone involved, then it becomes consensual. From such an explanation, it may be seen why persons with disabilities, as well as those with no disabilities can get confused with this subject of sex and sexuality. This is, therefore, the type of information needed by everyone to know, with or without a disability, to make sure that everyone understands the same thing when talking about sex and sexuality. Without such important information, we have unfortunate cases of sexual abuse of persons with disabilities, with perpetrators getting away with murder. People with disabilities also need proper understanding of sex and sexuality since what these terms may mean to one person can be totally different from another person's interpretation.

One thing for certain is that the above scenario will not even spare those going to school. Some schools have been known exempting learners with disabilities from sex education classes, because the school authorities consider such a subject irrelevant to persons with disabilities. Because of such exclusion, achievement of sexual identity will be very difficult for children with disabilities, especially those with intellectual challenges, who will forever remain ignorant about issues related to sexuality.

Specifically referring to the psycho-sociological perspective, sexuality has been understood as an expression of intimacy affection and love, virtues which may culminate in marriage. Having a disability or not: love, affection and intimacy are generally known to be the basis of marriage to all sexual human beings. Marriage, as defined by Henslin (2002) in Mutasa and Tafangombe (2010), is a group's approved mating arrangement marked out by a ritual, culturally solemnized by payment of lobola (bridal price). Since marriage is a mating arrangement approved by a group, cultural norms have to be socially channeled with full approval of all, inclusive of members of the extended family, like: grandparents, aunts, uncles, cousins and the nuclear family itself (Mutasa and Tafangombe 2010), if the exo-systematic setting is anything to go by. This being the case, therefore, almost all societies take marriage ceremonies very seriously.

To emphasize how crucial marriage ceremonies are culturally, there are practical incidences where prospective couple's marriages are disapproved and completely stopped, especially if the woman (bride to be) had a disability. In such communities, marriages of this nature are considered a cultural taboo and hence lamentable. Such a union is viewed as an abomination since it rubs against cultural and social ethos. Cancellation of such lobola ceremonies would be made, not even by the nuclear family, but members of the extended family who always happened to be the most vocal in such gatherings or events. Facts that culminated to this objection were based on the cultural belief that if such a union would be solemnized, a disability would run in the couple's family, as they raise their own children. So, stopping of such marriages was based on the premise that as long as one is living with a disability, they cannot exercise their sexual function, especially for procreation. For that reason, such a union would be considered a rejection of culture and tradition. The whole set up would even be further worsened if the man (bridegroom to be) was able bodied, yet things would even be different if it was the man who had a disability: Ridiculous isn't it? From the above incident, Majaya (2007) of the Jairos Jiri Centre for persons with disabilities in Zimbabwe, is quoted by Mutasa and Tafangombe (2010:140) as saying, “...it was easier for a disabled man to marry an able bodied woman, than for a disabled woman to marry an able bodied man..." 
Since growth and physical maturity are also known to be frameworks for understanding sexuality, according to Mutasa and Tafangombe (2010), studies have also shown that persons with disabilities also face problems of maturation, in the same way those with no disabilities do. The subject of maturation of persons with disabilities has also received considerable silence in some communities, resulting in these people experiencing inconsistencies with knowledge to do with physical growth and social maturation. As an offshoot, Chakuchichi et al (ibid) eventually see a physically mature woman in a young socially immature girl. As an aftermath, the family continues considering such a girl as a baby, when in actual fact she has to be treated as a young adult. Because of maturation inconsistencies, we have it on good record that some physically mature adolescence with disabilities, still sleep together with their parents, yet they were old enough to sleep in their own separate rooms. One reason to this scenario is that while a person with a disability may already be physically mature, for example, that exclusion from social activities with peers makes them remain socially immature.

\section{CONCLUSION}

This paper has made an attempt to show that issues to do with sexuality go beyond sex organs (genitalia) or bedroom activities. If anything, these issues also cover matters of sex drive, sex acts, and all those aspects of personality, concerned with learned communication and relationship patterns. It also emerged that sexuality and sexual realities of persons with disabilities have traditionally been given very little recognition, although it has proved to be an area of concern to every human being, including those with disabilities. Myths and taboos surrounding the subject have been noted as reasons enough for not involving persons with disabilities in sex and sexuality related discussions or debates, yet there are more similarities than differences in terms of sexual stimuli, sexual interests and deep seated feelings, which are commonly provided through personal interchanges. Although certain disabilities may impose limitations, the paper has shown that sex drive, sex acts and sexuality remain intact in persons with disabilities, as they are in those with no disabilities. Notwithstanding the above, some cultural beliefs, in some societies, are not only prohibitory and/or inhibitory to inclusion of persons with disabilities, but are regrettably grossly discriminatory and exclusionary in every sense. Being sexual beings also, persons with disabilities equally need to be properly informed about matters to do with sex and sexuality, in terms of: roles, patterns, desires, etc., since such define their whole beings with regards to sexual habits and feelings, towards same and the opposite sex. Since issues of sexuality are also characterised by interpersonal relationships, most of such information has to also come through friends and peers by social cohesion.

\section{REFERENCES}

[1]. Apfelbaum, B. (1989). Principles and Practice of Sex Therapy (2nd Ed), S. R. Lieblum \& L.A. Pervin (Eds), Guilford Press: New York.

[2]. Arnot, M. and Weiner, G. (1987). Gender under Scrutiny. London: Hutchinson.

[3]. Barron, R.D. and Norris, G.M. (1998). Sexuality Divisions and the Dual Labour Market. London: Macmillan.

[4]. Bernard, J. (2005). The Future of Marriage. Harmondworth: Penguin.

[5]. Chakuchichi, D.D., Mapepa, P. and Mutasa, J. (2010). Life Skills and Support Services for People with Disabilities. Zimbabwe Open University. Module MSE 505. Harare: ZOU.

[6]. Firestone, S. (2001). Dialectic of Sex. London: Paladin Publishers.

[7]. Haralambos, M. and Holborn, M. (1990). Sociology: Themes and Perspectives. London: Unwin Hyman Publishers.

[8]. Marinelli, R.P. and DellOrto, A.E. (1984). The Psychological \& Social Impact of Physical Disability. New York: Springer Publishing Company.

[9]. Mutasa, M. and Tafangombe, J. (2010). Disability in the Social Context. Zimbabwe Open University. Module MSE 603. Harare 2011.

[10]. Sexual Resource Center for Parents (SRCP)(2017) http://www.srcp.org/for_all_parents/definition.html).

[11]. Corinna, H. (Undated). "What's Sex?". www.scarleteen.com. 